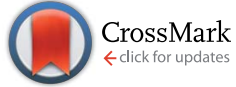

Cite this: RSC Adv., 2016, 6, 54754

Received 4th May 2016

Accepted 26th May 2016

DOI: 10.1039/c6ra11563b

www.rsc.org/advances

\section{Microcasting with agarose gel via degassed polydimethylsiloxane molds for repellency-guided cell patterning $\dagger$}

\author{
Nobuyuki Tanaka, ${ }^{a}$ Hiroyuki Moriguchi, ${ }^{a}$ Asako Sato, ${ }^{a}$ Takayuki Kawai, ${ }^{\text {ab }}$ \\ Kenta Shimba, ${ }^{c}$ Yasuhiko Jimbo ${ }^{c}$ and Yo Tanaka*a
}

\begin{abstract}
Hydrogel patterning methods are widely used for cell patterning because they offer better long-term stability than protein patterning methods such as micro-contact printing, but conventional hydrogel patterning methods require special apparatuses such as a laser or an electron beam lithography system or they have complicated chemical operations which prevent their practical use in biological laboratories. A simple method was developed to cast a hydrogel solution without external power sources using a polydimethylsiloxane (PDMS) mold with micro-channels. This study employed "the accumulation of vacuum pressure" in a degassed lump of PDMS as a driving force for introducing agarose solution into the micro-channels. Sufficient vacuum pressure could be accumulated within $1 \mathrm{~h}$ in the PDMS elastomer that was acting as a vacuum tank, and $2 \mathrm{w} / \mathrm{v} \%$ agarose solution could be aspirated into the micro-channels with widths from 100 to $2000 \mu \mathrm{m}$ and a height of $19 \mu \mathrm{m}$, fully filling them. After the gelation and dehydration of agarose solution in the micro-channels, the patterns of agarose gel on the channels were successfully cast with a 90\%-width accuracy. By using the repellency of agarose gel toward cell adhesion, patterned cultures of myoblasts and cortical neurons were successfully prepared. This technique is expected to be useful in repellency-guided cell patterning for various types of cells, with applications to cell-cell interactions and axon guidance.
\end{abstract}

\section{Introduction}

Tissue formation is one of most fundamental topics in multicellular systems and tissue engineering research. ${ }^{1-4}$ To investigate the relationship between tissue morphology and the biological phenomena of tissue composed of adherent cells, there has been a strong demand for a method to control tissue shape and cellular allocation, so-called cellular patterning. Recently, such patterning methodologies have reached the phase of practical use by biological researchers in various fields. Therefore, flexibility to form diverse shapes and realize single cell level resolution is the next major requirement to be met.

The fundamental concept of cell patterning is to locally divide cell culture substrates into cell adhesive and non-cell adhesive surfaces. One of the most popular methods of surface patterning is micro-contact printing. ${ }^{5-8}$ This is a simple method to pattern

${ }^{a}$ Quantitative Biology Center (QBiC), RIKEN. 1-3 Yamadaoka, Suita, Osaka 565-0871, Japan. E-mail: yo.tanaka@riken.jp

bJapan Science and Technology Agency, PRESTO, 4-1-8, Honcho, Kawaguchi-shi, Saitama 332-0012, Japan

${ }^{c}$ Department of Precision Engineering, School of Engineering, The University of Tokyo, Hongo 7-3-1, Bunkyo-ku, Tokyo 113-8656, Japan

$\dagger$ Electronic supplementary information (ESI) available. See DOI: $10.1039 / \mathrm{c} 6 \mathrm{ra11563b}$ molecules by contacting polydimethylsiloxane (PDMS) molds with a substrate and the patterning resolution has now reached the sub-micron level. A similar concept, protein patterning using striped PDMS micro-fluidic channels ${ }^{9}$ or using laminar flow, ${ }^{\mathbf{1 0}}$ has been reported. PDMS is commonly used in these methods because PDMS molds are naturally inert and they allow simple and easy fabrication of microchannels. However, these adhesion-protein-based partial surface modification methods have a problem with the longterm stability of the cell patterning.

On the other hand, hydrogel based surface control methods are known to have long-term stability. The hydrogel based methods, which are negative patterning methods, are based on the spatial distribution of repellency to cells by such materials as poly(ethyleneglycol), ${ }^{11}$ polyelectrolyte, ${ }^{8}$ phospholipid-likestructured polymer (2-methacryloyloxyethyl phosphorylcholine; MCP), ${ }^{12}$ polyacrylamide, ${ }^{13}$ and hydrophilic glycoprotein mucin, ${ }^{\mathbf{1 4}}$ In the case of negative patterning methods, no molecules for cell adhesion (e.g. fibronectin) are required. Those molecules tend to affect not only cell adhesion but also other cellular behaviors such as differentiation. ${ }^{\mathbf{1 6}}$ Therefore, hydrogel based patterning methods are quite important for distinguishing between morphological and biomolecular effects on cellular behaviors in some cellular patterning experiments such as in the mechanobiology field. ${ }^{\mathbf{1 5 , 1 6}}$ However, the methods to realize 
hydrogel based negative patterning such as hydrogel coating using an electron beam, ${ }^{17}$ inkjet printer, ${ }^{18}$ excimer laser, ${ }^{19} \mathrm{UV}$ light, ${ }^{12}$ or IR laser ${ }^{20}$ require a dedicated apparatus or complicated chemical operations. By contrast, PDMS stencil based molding, ${ }^{21,22}$ mechanical fabrication, and 3D printer based molding are rather easy to use, but their resolution is low (approximately $50 \mu \mathrm{m}$ or more). This fact prevents their practical use for biological research.

Here, a completely different approach was proposed that uses PDMS micro-fluidic channels as a mold for negative patterning hydrogel casting. This study used agarose as the hydrogel. Agarose is an extremely inert substance with respect to cellular adhesion ${ }^{18}$ and biomolecular absorption, ${ }^{23}$ and it is widely available in biological laboratories as a basic material for various purposes such as agarose gel electrophoresis, ${ }^{24} \mathrm{E}$. coli culturing on agarose culture media, ${ }^{25}$ and agarose gelembedded cell culturing. ${ }^{26}$ Essentially, surface modification can be done with agarose in a quite simple procedure with temperature control to change the agarose from a solution to a gel, followed by dehydration of the agarose gel. ${ }^{27}$ Furthermore, agarose is safely used without the limitations needed for toxic substances such as acrylamide. ${ }^{28,29}$ In addition to these advantages, PDMS molds are easy to fabricate, even without complicated equipment in biological laboratories, for individual cell culture experiments if master molds are prepared. The proposed method is especially suitable for patterning cultures in large areas or in curved structures. Since the hydrogel casting method is based on photolithography, the resolution is approximately $1 \mu \mathrm{m}$. Therefore, the new micro-casting method combines the advantages of protein patterning and hydrogel patterning methods to achieve simple use, long-term stability and high resolution.

Based on this background, the objective of this report was to demonstrate agarose patterning using PDMS molds and apply it to actual cell culture experiments. An obstacle in the use of agarose for micro-patterning is its high viscosity and low wetting characteristics toward PDMS, and these characteristics prevent agarose solution from penetrating into micro-channels made of PDMS. ${ }^{30}$ Therefore, this study proposed the idea to use vacuum pressure accumulated in PDMS elastomer to pump agarose solution into the microchannels. This study developed a method for realizing cellular patterning on cell culturing surfaces with a micro-cast agarose gel. Although hardly any agarose solution can be introduced into micro-channels on a cell culturing surface because of its high viscosity, this study succeeded in filling the micro-channels with the viscous agarose solution by using vacuum pressure that remained in empty spaces of PDMS surrounding the micro-channels after degassing (Fig. 1A-E). After removal of the PDMS micro-channels from the dish culturing surface, the micro-cast agarose patterns were obtained on the surface (Fig. 1F). When a different micro-mold having micro-columns at specific locations was used, cast agarose patterns having punched-out holes exposing the bare dish surface could be obtained (Fig. 1G and H). Because agarose gel has a high repellency for cell adhesion, cells

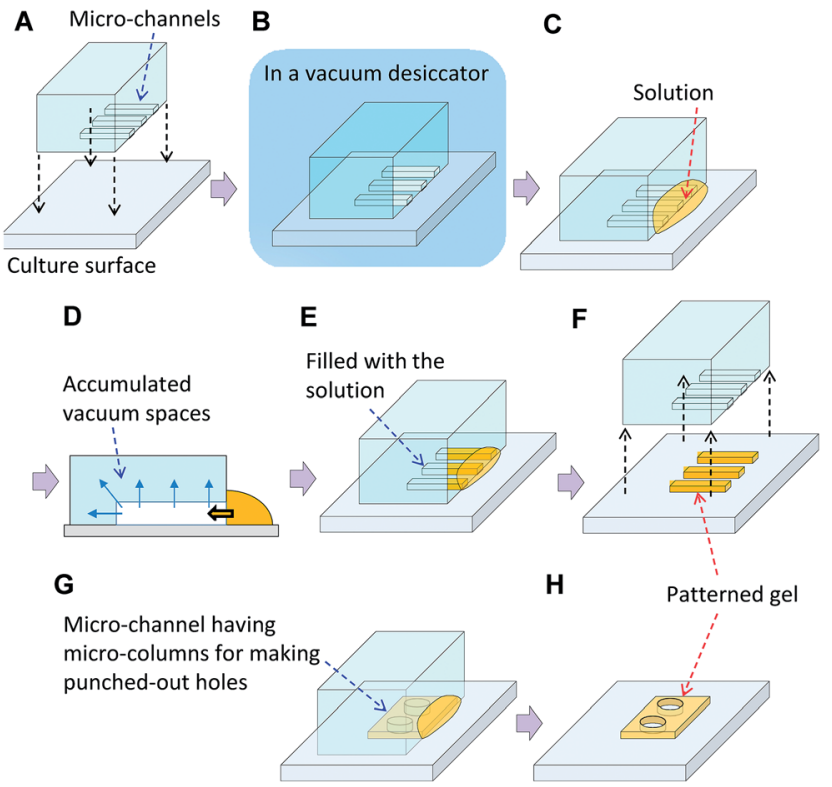

Fig. 1 Microcasting with agarose gel. (A) A PDMS mold with microchannels was placed on a dish culturing surface. (B) The culturing surface and mold were degassed in vacuum for $1 \mathrm{~h}$. (C) After degassing, gel solution was immediately supplied at one end of the microchannels. (D) The solution was sucked into the micro-channels by the vacuum pressure accumulated in the mold. (E) After gelation of the solution, the dehydration of gel was done. (F) Next, the mold was carefully removed from the culturing surface, resulting in microchannel patterns on the dish. (G) A PDMS mold having micro-columns for making punched-out holes could also be used. $(\mathrm{H})$ The resulting pattern had punched-out holes where the bottoms exposed the bare surface of the dish.

adhered only at the non-agarose-covered areas of the cell culture dish after cell seeding.

\section{Materials and methods}

\section{Fabrication of PDMS micro-channels}

PDMS micro-channels were fabricated by a photolithographic method described in previous reports ${ }^{6,7}$ with some modifications. Briefly, negative photoresist (SU-8 2035) (Nippon Kayaku, Tokyo) was coated on a 2-inch-diameter Si-wafer (p-type, mirror-finished $\langle 100\rangle$ surface) (SEMITEC, Chiba) with a spin-coater (1H-D7) (Mikasa, Tokyo) spinning first at $500 \mathrm{rpm}$ for $10 \mathrm{~s}$ and then at $4500 \mathrm{rpm}$ for $30 \mathrm{~s}$ more. The photoresist covering the Si-wafer was baked at $95{ }^{\circ} \mathrm{C}$ for 10 $\mathrm{min}$. The photoresist was exposed to UV light at an intensity of $9.9 \times 10^{3} \mathrm{~mW} \mathrm{~cm}^{-2}$ for $12 \mathrm{~s}$ with a mask aligner (MA-10) (Mikasa) through a photomask having a desired pattern. The photoresist after UV-exposure was baked at $95{ }^{\circ} \mathrm{C}$ for 3 min and treated with a developer, 2-methocy-1-methylethyl acetate (130-10505) (Wako, Osaka). The remaining photoresist on the wafer was used as a mold for the micro-channels. PDMS and a curing agent for PDMS (Sylpot $184 \mathrm{~W} / \mathrm{C}$ ) (Dow Corning Toray, Tokyo) were mixed at a ratio of 10 to 1 . The PDMS mixture was poured into the Si-wafer mold and cured at 
$80{ }^{\circ} \mathrm{C}$ for $3 \mathrm{~h}$. The cured PDMS was peeled off from the Si-wafer and trimmed, resulting in a micro-channel mold for the gel solution.

\section{Agarose gel casting with micro-channels}

The surface of the PDMS mold was cleaned with mending tape (MP-18) (Sumitomo 3M, Tokyo, Japan). After being rinsed with ethanol, the PDMS mold with micro-channels on a tissue culture polystyrene (TCPS) dish (Falcon 353003) (Corning, Inc., Corning, NY) was placed in a vacuum desiccator (VL-C) (ASONE, Osaka) that was connected to a vacuum line at a gauge pressure of $-98 \mathrm{kPa}$ for $1 \mathrm{~h}$ degassing. Immediately after stopping the vacuum application, the mold and dish were removed from the desiccator and $2 \mathrm{w} / \mathrm{v} \%$ agarose solution (A2576) (Sigma-Aldrich, St. Louis, MO) heated to boiling in a microwave oven was poured onto one end of the PDMS micro-channels (Fig. 1C). The behavior of the solution was monitored with a digital movie camera (HDR-CX560) (SONY, Tokyo). After the micro-channels were filled with the solution, it was cooled at r.t. for $30 \mathrm{~min}$ to get agarose gelation. Next, the gelated solution was dehydrated in a drying oven (EO-300B) (ASONE) at $70{ }^{\circ} \mathrm{C}$ for $30 \mathrm{~min}$. The dehydrated agarose patterns were harvested by peeling only the PDMS micro-channels from the dish surface (Fig. 1F). The PDMS molds could be repeatedly used by cleaning their surfaces after each use as described above.

\section{Viscosity, surface tension, and contact angle measurement}

The viscosity of $2 \mathrm{w} / \mathrm{v} \%$ agarose solution was measured with a viscosity meter (SV-10) (A\&D, Tokyo). The surface tension of 2 $\mathrm{w} / \mathrm{v} \%$ agarose solution was measured by a pendant drop method. ${ }^{31}$ The contact angles of $2 \mathrm{w} / \mathrm{v} \%$ agarose solution against PDMS, TCPS, and glass slide (S9213) (Matsunami, Osaka) surfaces were measured. The plates of these materials were warmed on a hot plate at four different temperatures of 25, 50, 70 , and $90^{\circ} \mathrm{C}$ and a $20 \mu \mathrm{L}$ droplet of agarose solution, heated to boiling in a microwave oven, was dropped onto them. The droplet that adhered on the surface was monitored with a digital camera (Nikon 1 V1) (Nikon, Tokyo). The digital image was shown on a personal computer screen and the contact angle of the droplet was determined from the image using drop shape analysis software. ${ }^{32}$

\section{Vacuum pressure measurement}

Several-millimeter-thick PDMS sheets with a weight of $106 \mathrm{~g}$ were plated in a homemade pressure container, which was equipped with a digital manometer for gauge pressure (KDM30) (Krone, Tokyo), a vacuum line with a valve, and a release valve to the atmosphere. The container volume was $338 \mathrm{~mL}$ and the PDMS sheets had a total volume of $103 \mathrm{~mL}$.

The measurement was done in the following steps; (1) applying a vacuum at $-98 \mathrm{kPa}$ for $1 \mathrm{~h},(2)$ releasing the vacuum at $0 \mathrm{kPa}$ and immediately closing the valve, and (3) monitoring the gauge pressure value of the manometer.

The time constant of the pressure descent was determined from the monitored values of gauge pressure.

\section{Dimensional analysis}

The surface profiles of SU-8, PDMS micro-channels, and patterned dehydrated agarose were measured with a threedimensional laser scanning confocal microscope (VK-8710) (Keyence, Osaka).

\section{Patterned cell culture}

Cells were maintained by a standard protocol for the individual strains of cells. ${ }^{33,34}$ Mouse skeletal myoblasts (C2C12) were maintained in Dulbecco's modified Eagle's medium (D6429) (Sigma-Aldrich) supplied with $10 \mathrm{v} / \mathrm{v} \%$ fetal bovine serum (Nichirei Biosciences, Tokyo) and $1 \mathrm{v} / \mathrm{v} \%$ penicillin-streptomycin (168-23191) (Wako). C2C12 cells were passaged by a trypsin-digest method before full-confluency. After the dehydrated agarose patterns were fabricated on the culture dishes, C2C12 cells were seeded onto the dishes at an initial density of 5 $\times 10^{4}$ cells per $\mathrm{cm}^{2}$. The cells were cultured at $37{ }^{\circ} \mathrm{C}$ in a humidified condition with $5 \% \mathrm{CO}_{2}$.

This research was performed in accordance with the University of Tokyo's guidelines regarding animal research and the following procedure for preparing cortical cells was approved by the ethics committee of the University of Tokyo (B12-04, KA-14-1). Cortical tissues were dissected from E16 ICR mouse embryos (Charles River Laboratories Japan, Yokohama) and dissociated with 0.25 w/v\% trypsin (Thermo Fisher Scientific, Waltham, MA) and $10 \mathrm{mg} \mathrm{mL}^{-1}$ DNase (Sigma-Aldrich). Cortical cells were plated onto the agarose patterns at an initial density of $3 \times 10^{4}$ cells per $\mathrm{cm}^{2}$. The cells were maintained in Neurobasal ${ }^{\circledR}$ medium supplemented with $2.5 \mathrm{mM}$ GlutaMAX ${ }^{\mathrm{TM}}$, a set of B-27 supplements, and $100 \mathrm{U} \mathrm{mL}^{-1}$ to 100 $\mathrm{mg} \mathrm{mL}^{-1}$ penicillin-streptomycin (all from Thermo Fisher Scientific) at $37{ }^{\circ} \mathrm{C}$ in a humidified condition with $5 \% \mathrm{CO}_{2}$. Half of the medium was replaced every three days.

\section{Results and discussion}

\section{Agarose gel casting}

Agarose solution supplied at one end of the micro-channels never moved into the micro-channels of PDMS when there was no degassing during a $30 \mathrm{~min}$ observation (Fig. 2A); however, the degassed PDMS micro-channels was able to draw in the agarose solution (Fig. 2B). The time to fill the $19 \mu \mathrm{m}$-high and $19 \mathrm{~mm}$-long micro-channels with agarose solution was from 20 to $100 \mathrm{~s}$ when increasing the width of the microchannels from 100 to $2000 \mu \mathrm{m}$ (ESI Movie $1 \dagger$ ). Because the PDMS channels were completely filled with agarose and then the gelation occurred, even in the slowest case of agarose filling, the filling was sufficiently quicker than the gelation. The flow rate of agarose solution was determined to be from 1.7 to $7.7 \mathrm{~nL}$ $\mathrm{s}^{-1}$ for 100 and $2000 \mu \mathrm{m}$-width micro-channels, respectively (Fig. 2C). When agarose patterns having punched-out holes are fabricated (Fig. $1 \mathrm{G}$ and $\mathrm{H}$ ), these patterns should be surrounding larger agarose areas with widths from $100 \mu \mathrm{m}$ to millimeter order, especially in the case of large-area casting. Therefore, the value of the flow rate would be useful for estimating the time of agarose penetration. The relationship 


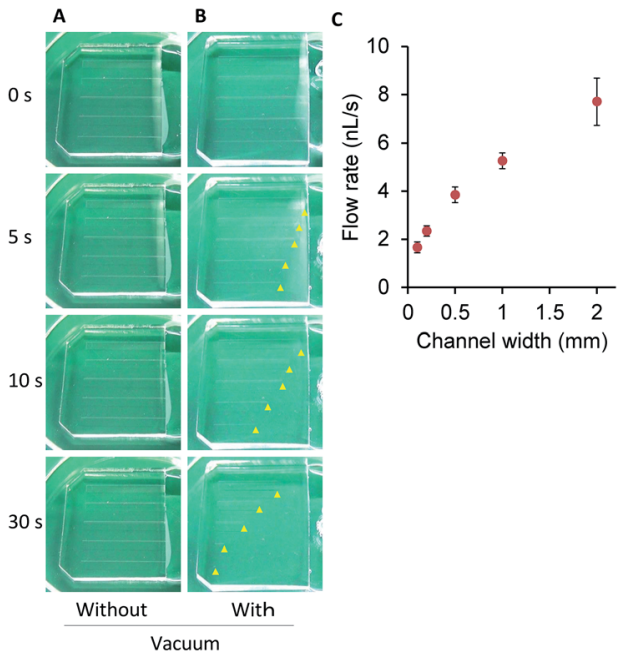

Fig. 2 Vacuum-assisted filling of agarose solution into micro-channels. Columns of photographs (A) and (B) show the appearance of agarose solution at $0,5,10$, and $30 \mathrm{~s}$ after supplying the solution at the end of $19 \mathrm{~mm}$-length micro-channels of PDMS molds without and with accumulated vacuum pressure, respectively. Yellow arrowheads indicate points in each micro-channel where agarose solution had penetrated at the times the photos were taken. Graph (C) shows the relationship between the flow rate of agarose solution and width of the micro-channels. The closed circles and error bars indicate the average values and standard deviations, respectively $(n=4)$.

between the flow rate and width was almost linear for widths from 500 to $2000 \mu \mathrm{m}$. However, at widths less than $500 \mu \mathrm{m}$, the flow rates decreased steeply compared with those over $500 \mu \mathrm{m}$. Pressures acting on the agarose solution in the micro-channels were thought to be (1) capillary pressure, (2) pressure loss caused by fluidic viscosity, and (3) vacuum pressure in the PDMS forming the channels.

To assess capillary pressure, the interfacial properties of agarose solution against three different types of materials PDMS, TCPS, and glass were assessed by the measurement of contact angles (Fig. 3A-C). Although the capillary force is generated by the surface tension of solution at the interface between the liquid and the wall of the micro-channel, the direction of the force is determined by the cosine direction of the contact angle. The pressure applied on the interface $p_{\mathrm{c}}$, driving a capillary pressure, is given by the following equation, ${ }^{35}$

$$
p_{\mathrm{c}}=\cos \theta_{\mathrm{c}} \gamma\left(\frac{2}{w}+\frac{1}{h}\right)+\cos \theta_{\mathrm{b}} \gamma \frac{1}{h}
$$

where $\theta_{\mathrm{c}}$ is the contact angle (CA) of the micro-channel; $\theta_{\mathrm{b}}$, CA of the bottom; $\gamma$, surface tension of the solution; $h$, height of the micro-channel and $w$, width of the micro-channel. Eqn (1) indicates that the surface tension acts as an intrusivedirectional force, which means there is a potential to fill the micro-channels with solution when the contact angle is less than $90^{\circ}$. The contact angles of agarose solution were less than $90^{\circ}$ at 25 to $90^{\circ} \mathrm{C}$ for TPCS and glass surfaces, and for the PDMS surface at $90^{\circ} \mathrm{C}$. The result suggested that the surface tension of agarose solution was able to produce an intrusive-directional force for all the materials at $90{ }^{\circ} \mathrm{C}$. Therefore, at a no-fluidic-
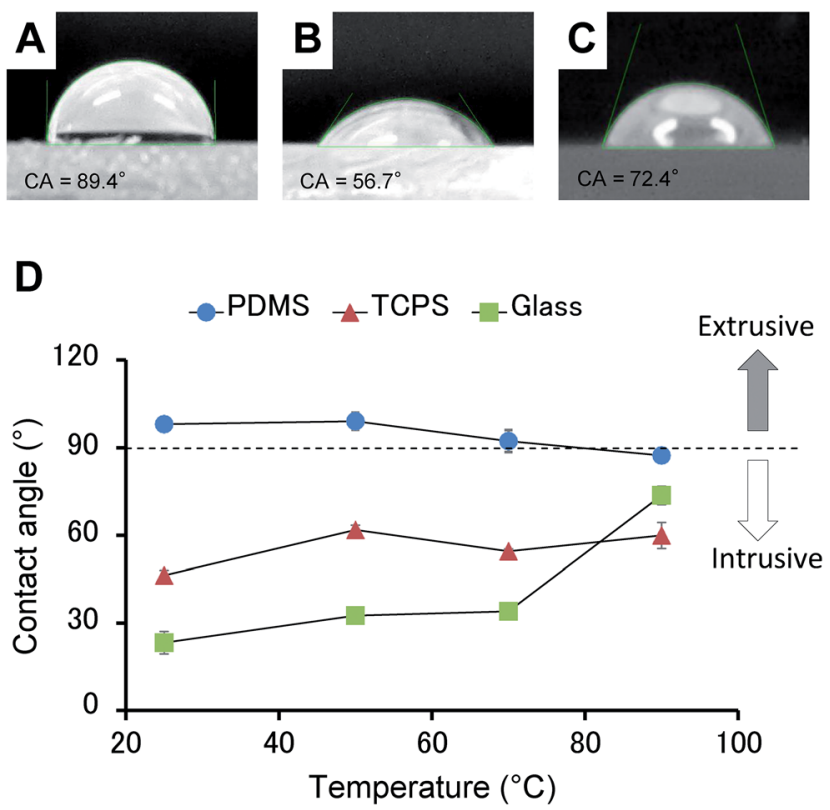

Fig. 3 Contact angle (CA) analysis of agarose solution. Photographs $(A-C)$ show droplets of agarose solution at $90^{\circ} \mathrm{C}$ adhered on three different types of surfaces: (A) PDMS, (B) TCPS, and (C) glass. Graph (D) shows CA of agarose solution for the surfaces at temperatures of 25 , 50,70 , and $90^{\circ} \mathrm{C}$. The data markers and error bars indicate average values and standard deviations, respectively $(n=5)$.

resistance condition, the micro-channels were expected to be spontaneously filled with the solution as long as the temperature of solution and channel surface was higher than $90{ }^{\circ} \mathrm{C}$. Since the measured value of surface tension $(\gamma)$ of agarose solution was $60.1 \mathrm{mN} \mathrm{m}^{-1}$, the capillary pressure $p_{\mathrm{c}}$ was estimated and its maximum value reached $2.4 \mathrm{kPa}$ in a $20 \mu \mathrm{m}$-high
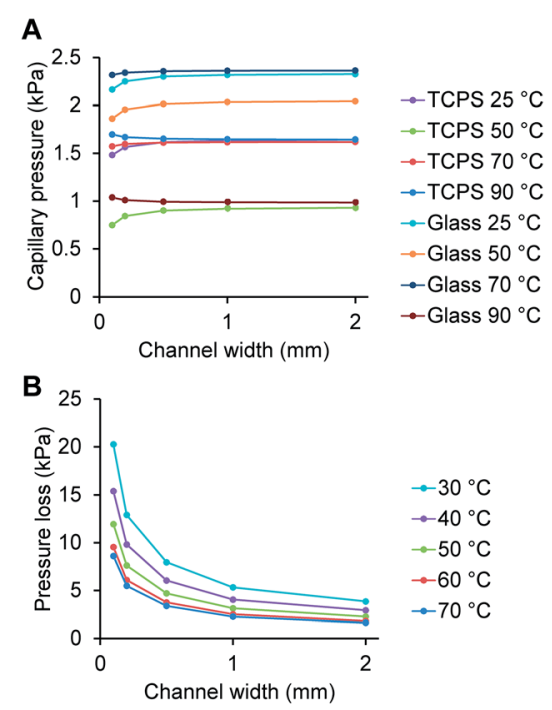

Fig. 4 Changes in the capillary pressure (A) caused by the surface tension between agarose solution and two basal materials, TCPS and glass. Changes in the pressure loss (B) caused by the fluidic viscous resistance at various temperatures. 


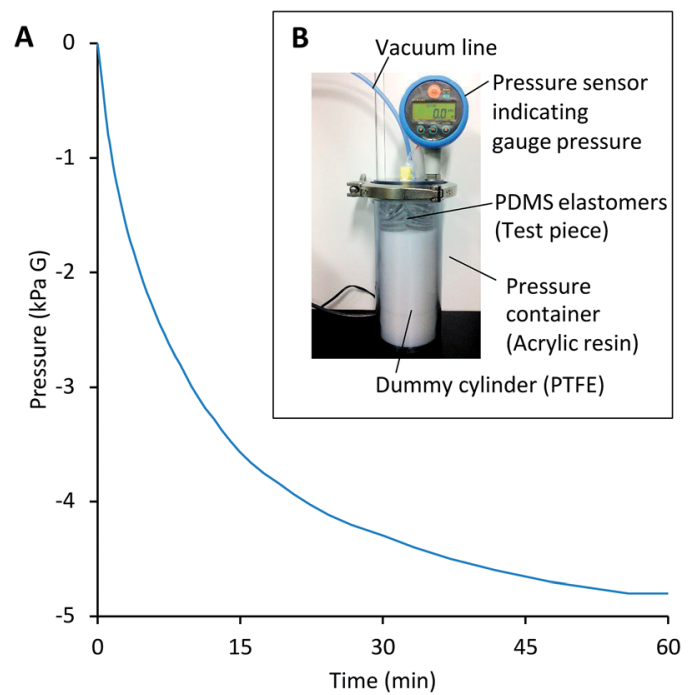

Fig. 5 Vacuum pressure remaining in PDMS elastomer. Graph (A) shows the time-course of gauge pressure measured by the experimental setup shown in photograph (B).
PDMS micro-channel with a width of $2000 \mu \mathrm{m}$ on a glass surface at $50{ }^{\circ} \mathrm{C}$ (Fig. 4A). All the values were positive, indicating that an intrusive direction pressure appeared in the micro-channels, and all pressure values were almost constant regardless of the widths, therefore, the capillary pressure was not the reason why the flow rate decreased in the narrow channels with a width of less than $500 \mu \mathrm{m}$.

The relationship between pressure loss $\Delta p$ caused by fluidic viscous friction and flow rate $q$ was derived from the HagenPoiseuille law as the following equation, ${ }^{35}$

$$
\Delta p=12 \eta \frac{(w+h)^{2} L}{(w h)^{3}\left(1+\frac{5 h}{6 w}\right)} q
$$

where $\eta$ and $L$ are the viscosity of fluid and the length of fluidfilled channel, respectively. Pressure loss $\Delta p$ was estimated (Fig. 4B) under an actual condition with a value of $\eta=34.6 \mathrm{mPa}$ $\mathrm{s}$ for $2 \mathrm{w} / \mathrm{v} \%$ agarose solution at $30{ }^{\circ} \mathrm{C}$. The value of pressure loss $\Delta p$ was quite high at widths of 100 and $200 \mu \mathrm{m}$. Therefore, the main factor for decreasing the flow rate of agarose solution in the narrower micro-channels was speculated to be pressure loss
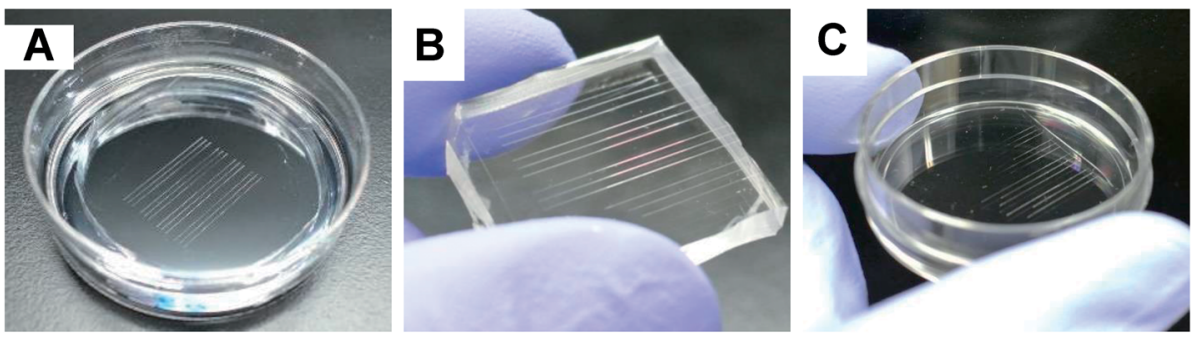

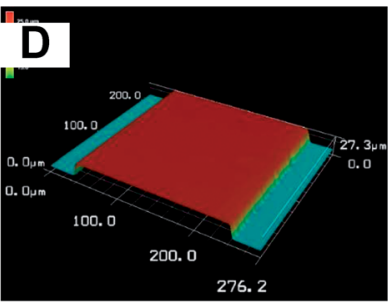

G

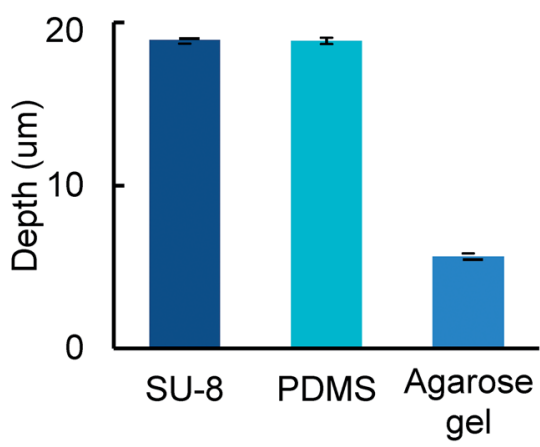

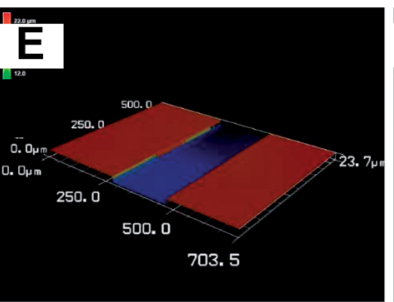

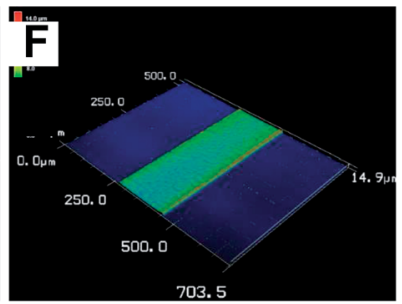

H

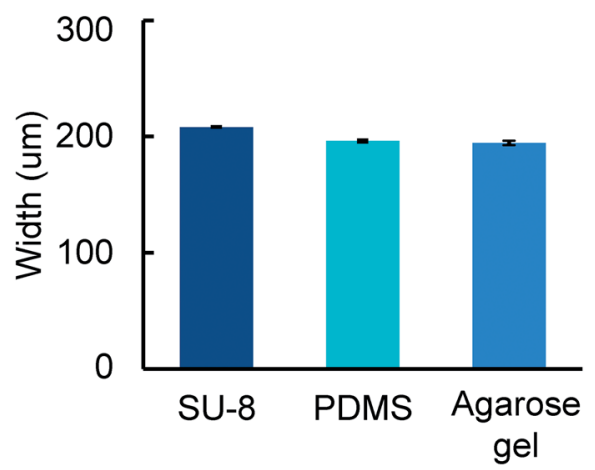

Fig. 6 Fabrication process using vacuum-assisted molding of agarose gel. (A) Fabrication of photoresist SU-8 convex patterns on Si wafer. (B) PDMS mold with micro-channel patterns that had been transferred from SU-8 patterns. (C) Agar-gel patterns on a cell culture dish. Threedimensional images (D)-(F) showing the surface profiles of SU-8, PDMS micro-channel, and agar-gel patterns, respectively. These photographs were taken through a confocal laser microscope. Graphs $(G)$ and $(H)$ show the depth and width of patterns, respectively. Column heights and error bars indicate the average values and standard deviations, respectively $(n=3)$. 
cause by the fluidic viscous friction. Sucking by the vacuum pressure accumulated in the PDMS elastomer allowed the agarose solution to penetrate into the micro-channels, even though the viscosity of agarose solution, which caused a high pressure loss in the narrower channels, was 30 times higher than that of water $(0.8 \mathrm{mPa})$.

To estimate the possible degree of vacuum pressure accumulated in PDMS elastomer, pieces of PDMS with a thickness of $2.8 \pm 0.49 \mathrm{~mm}$ (average $\pm \mathrm{SD}$ ) were put in a hermetically-sealed container, which was evacuated at $-98 \mathrm{kPa}$ G for $1 \mathrm{~h}$ (Fig. 5). After the vacuum was released at $0 \mathrm{kPa}$ G (atmospheric pressure), the valve was closed immediately, and the gauge pressure was monitored, and found to gradually decrease and to reach a plateau of $-4.8 \mathrm{kPa}$ within $60 \mathrm{~min}$ (Fig. 5A). The equivalent volume $V_{\text {eq }}$ to the vacuum pressure $p_{\mathrm{v}}$ of $-98 \mathrm{kPa}$, which corresponded to the degassing pressure, was calculated as based on Boyle's law

$$
p_{\mathrm{a}}\left(V_{\mathrm{c}}-V_{\mathrm{eq}}\right)+p_{\mathrm{v}} V_{\mathrm{eq}}=\left(p_{\mathrm{a}}-p_{\mathrm{ex}}\right) V_{\mathrm{c}}
$$

where $p_{\mathrm{a}}$ is atmospheric pressure; $p_{\mathrm{ex}}$, measured pressure in the container at the plateau; and $V_{\mathrm{c}}$, the container volume. The calculated equivalent volume $V_{\text {eq }}$ was $16.6 \mathrm{~mL}$ and equivalent to $16.1 \%$ volume of PDMS elastomer. The time constant of the pressure descent was $9.9 \mathrm{~min}$ assuming that the pressure changed logarithmically. This time-response of vacuum pressure was thought to be related to the flux of the vacuum via the PDMS surface and the value of the time constant was almost the same as that previously reported $(7.9 \mathrm{~min}) .{ }^{36}$ This result suggested that vacuum pressure-assisted agarose solution penetration into the micro-channels was performed within $10 \mathrm{~min}$ efficiently. Actually, the vacuum pressure accumulated in PDMS equilibrates to atmospheric pressure not only from the surface of the inner channel wall but also from the outer surface of the PDMS facing the ambient air such as the top and sides of the PDMS mold. The equilibration was delayed from the time at which atmospheric exposure was started, because the atmospheric gas molecules gradually penetrated into the degassed PDMS elastomer mold. Therefore, both inside and outer surfaces provided vacuum pressure. The benefit of the "aspiration-like process" was thought to be avoiding air bubbles from being trapped at a dead-end and at narrow corners of channels during agarose penetration in comparison with the positive pressure driving method where such trapping occurred.

\section{Evaluation of hydrogel patterning performance}

The photolithography method was used and surface profiles made by photoresist SU-8 on Si wafer were replicated onto the PDMS surface inversely, where a convex SU-8 structure on $\mathrm{Si}$ wafer became a concave PDMS profile (Fig. 6A and B). After the PDMS micro-channel mold was removed from the dish surface, the dehydrated agarose pattern of micro-channels was obtained on the surface (Fig. 6C). To understand how the dimensions were changed in the replication process between SU-8 and PDMS molds or the PDMS mold and cast agarose patterns, the SU-8 pattern on Si wafer, the PDMS micro-channel pattern, and the dehydrated agarose micro-channel pattern were observed with a microscope. The shapes of the SU-8 and agarose patterns were convex, and that of the PDMS micro-channels was concave (Fig. 6D-F). Because negative photoresist such as SU-8 remains only at the UV-exposed area, a photomask with the intact desired pattern of agarose should be prepared. Although the height of the SU-8 and the depth of the PDMS micro-channels were mostly identical, the height of the agarose patterns was significantly less than those of SU-8 and PDMS micro-channels by $30 \%$ (Fig. 6G). On the other hand, the widths of those profiles were slightly decreased in the order of SU-8, PDMS microchannels, and agarose patterns with a maximum variation of $8 \%$ (Fig. $6 \mathrm{H}$ ). Furthermore, the variations of depth and width were quite small within a maximum variation coefficient of $3 \%$ in the height of the agarose patterns. Basically, agarose gel shrinks during its dehydration. ${ }^{37}$ The results suggested that the dehydration process of agarose solution only affected the height of the agarose patterns. In the comparison of contact angle of agarose solution on PDMS, TCPS, and glass surfaces, the contact angles of agarose on the TCPS surface or the glass surface were smaller than $90^{\circ}$, on the other hand, those on the PDMS surface were over $90^{\circ}$ at 25 to $70{ }^{\circ} \mathrm{C}$ and larger than those on the TCPS surface or the glass surface (Fig. 3D). This result indicated that TCPS or glass had an affinity to agarose solution, PDMS had a weaker one than that of TCPS or glass, and PDMS repelled it at 25 to $70{ }^{\circ} \mathrm{C}$. For this condition, the agarose gel in a channel would become detached from the PDMS surface. Therefore, the agarose gel shrunk in height by losing contact with the PDMS. On the other side, because the agarose gel on the TCPS or glass surface kept contact with the surface, the gel was speculated to maintain its lateral dimension. Therefore,

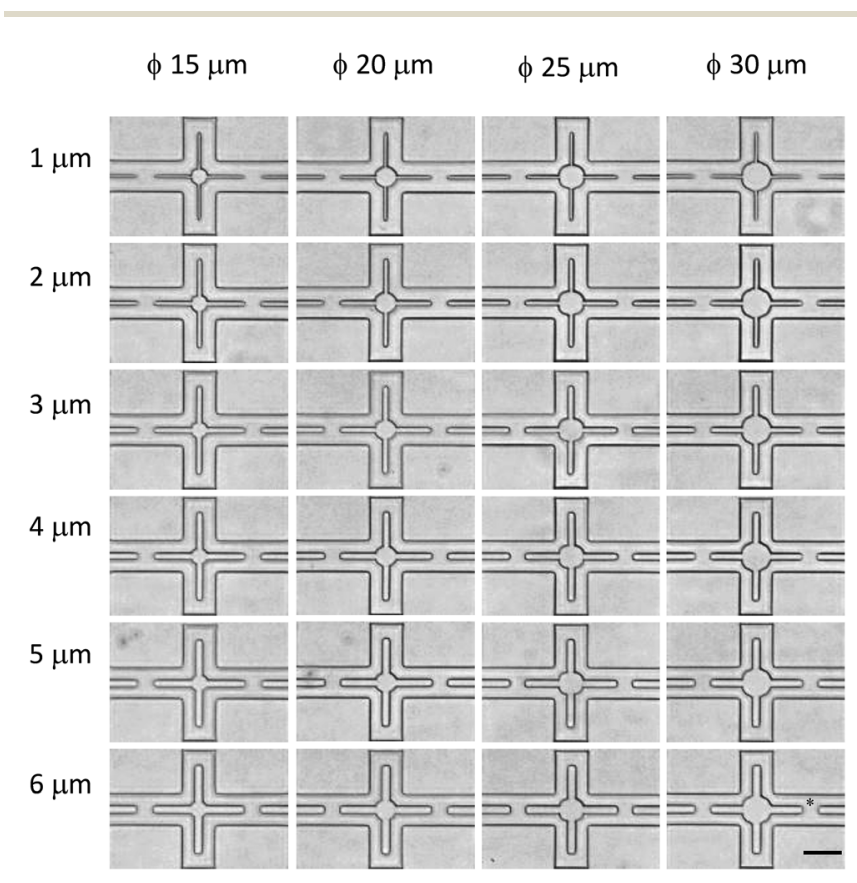

Fig. 7 Patterns of various dimensions produced by vacuum-assisted molding. The agarose area is indicated by an asterisk $(*)$ in the rightmost lowest panel. The black bar indicates $100 \mu \mathrm{m}$ and the scales of all panels are the same. 
this method would be useful for patterning viscous gel solutions such as agarose solution with a high accuracy in the horizontal dimension on glass and TCPS surfaces. The accuracy in the vertical dimension also could be improved by compensating for the shrinking of gel in the dehydration process.

As shown in Fig. 7, nearly $1 \mu \mathrm{m}$ resolution of the hydrogel patterning was achieved. This is because the developed method was based on photolithography and such resolution cannot be realized by a normal rapid prototyping apparatus (such as a 3D printer) or by mechanical processing. Even though a similar agarose patterning method was reported previously, ${ }^{30}$ the resolution was more than $10 \mu \mathrm{m}$, and various patterning shapes were not investigated. The degassing approach allowed introduction of hydrogel solution into micro-channels with various small features narrower than $20 \mu \mathrm{m}$, corresponding to the size of a single cell (ESI Fig. $1 \dagger$ ). Furthermore, agarose casting was possible on curved surfaces (ESI Fig. $2 \dagger$ ).

Although liquid introduction using PDMS degassing itself was reported previously, ${ }^{36,38}$ applying the technique to hydrogel molding and cell patterning as described in the following section is an original point of this study. The previous studies have applied fixed micro-channels, which were bonded to the bottom surfaces, to electrophoresis and immunoassays. Agarose casting for cell patterning requires non-bonding microchannels on the culturing surface, because the PDMS molds must be removed from the culturing surfaces to maintain intact cast agarose on the surfaces before cell culturing. This study found that the PDMS degassing approach was suitable for
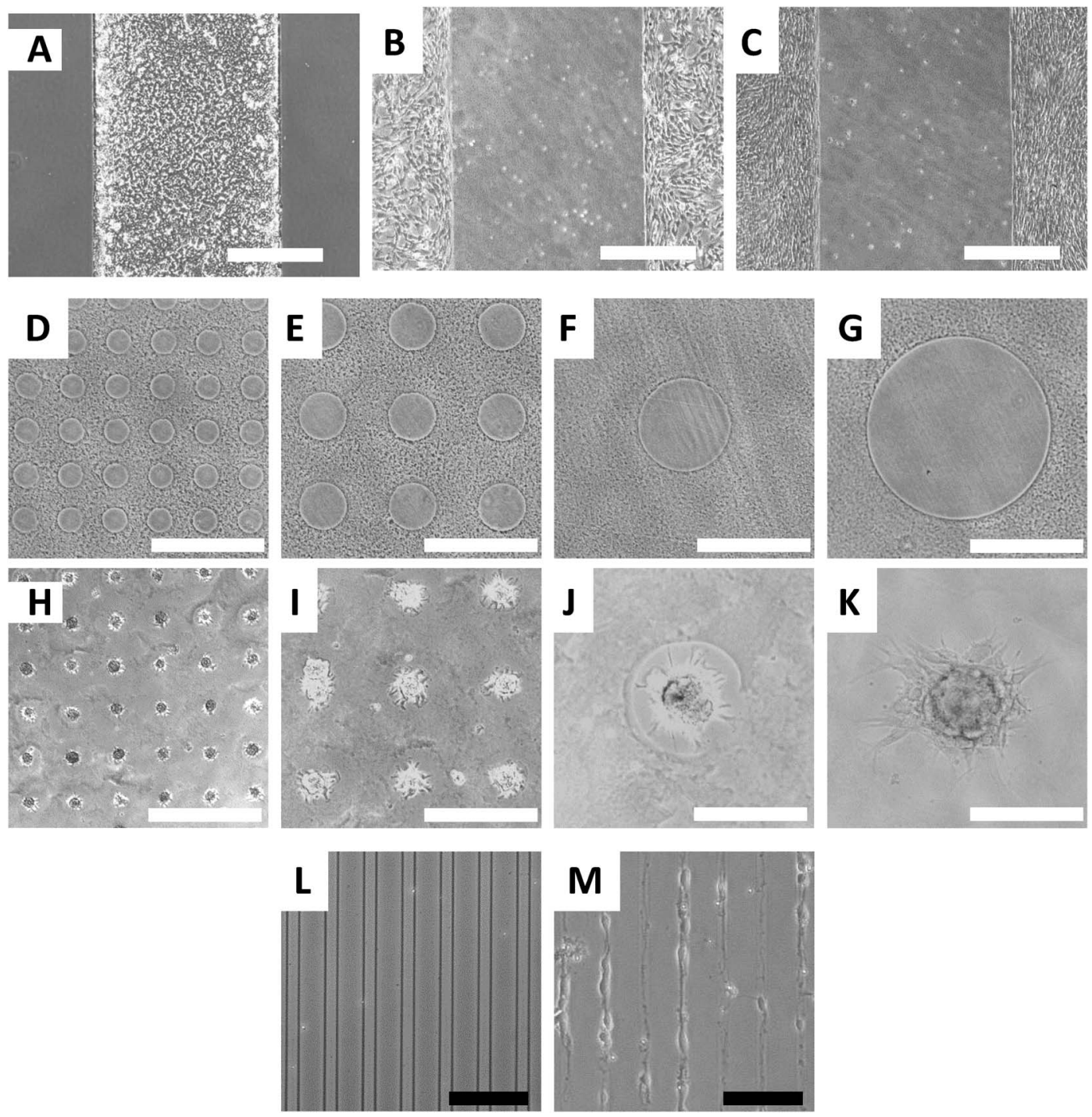

Fig. 8 Adhesion-area-regulated cell culture on vacuum-assisted molded agarose patterns. Microphotographs in the upper row show (A) agarose pattern and mouse skeletal myoblasts C2C12 cultured on the pattern at 1 day (B) and 2 days (C) after cell seeding. Microphotographs (DG) in the second row show 100-, 200-, 400-, and $800 \mu \mathrm{m}$-diameter circle agarose patterns without cells, respectively. Micrographs (H-K) show C2C12 cells cultured on the circle patterns corresponding to (D-G). Micrographs (L) and (M) show $50 \mu \mathrm{m}$-width agarose lanes with $33 \mu \mathrm{m}$-width bare-surface lanes and mouse cortical neuron cells cultured on the bare-surface lanes for 5 days. White and black bars indicate 500 and $100 \mu m$, respectively. 
pumping solution into non-bonding micro-channels, even for agarose solution which has a high viscosity, and this is something that previous studies have never mentioned.

\section{Micro-patterning of cells}

Mouse skeletal myoblasts C2C12 adhered on the bare surface of the agarose-micro-molded cell culturing dish at 1 day after cellseeding (Fig. 8A and B). Although the cells were observed to attach only on the bare-surface-exposed areas, no cells were found on agarose-molded areas. The cells grew to full confluency in the 2 day cultivation and maintained the initial adherent area where the cells never invaded the agarose area (Fig. 8C). These results suggested that the micro-cast agarose had a potential for micro-patterning cells as a negative patterning method based on the sufficient repellency of agarose toward adherent cells such as $\mathrm{C} 2 \mathrm{C} 12$. The micro-cast agarose can be maintained in the cell culture environment, because the dehydrated agarose is nearly insoluble in aqueous solution at 37 ${ }^{\circ} \mathrm{C}$, corresponding to the temperature of cell culture.

Circular punched-out holes in the agarose pattern were successfully obtained using PDMS molds with micro-columns in the channel with diameters of $100,200,400$, and $800 \mu \mathrm{m}$ (Fig. 8D-G). After a 2 day cultivation, C2C12 cells attached only in the circularly-bare-surface-exposed areas surrounded with agarose, and the aggregation of cells was observed. The aggregated $\mathrm{C} 2 \mathrm{C} 12$ cells were found to shrink to the center of a circular pattern where the diameter of the $\mathrm{C} 2 \mathrm{C} 12$ aggregation was decreased by $80 \%$ (Fig. $8 \mathrm{H}-\mathrm{K}$ ). Therefore, not only the top but also the side walls of agarose showed a repellent property to the cells. Furthermore, as compared with the straight pattern (Fig. 8A-C), the contractile force in the circular pattern was speculated to be stronger than that of the straight pattern where the force caused the cellular aggregation, because the contractile force in the circular pattern (the curvatures were positive) was concentrated into the center of the pattern.

This agarose micro-molding method could also be applied to mouse cortical neuron cells (Fig. 8L and M). The neurons were well aligned on the area between the agarose with a width of 17 $\mu \mathrm{m}$ at 5 days after cell seeding. In this condition, the shape of the neurons was elongated in the direction of striped patterns, and several axons extended from neurons along the striped patterns as previously reported. ${ }^{9}$

Those results suggested that micro-cast agarose patterns had a high potential to regulate cellular patterns for the various types of cells from highly proliferative $\mathrm{C} 2 \mathrm{C} 12$ cells to lowly proliferative neurons even for a 5 day cultivation. Therefore, it is believed that agarose is an appropriate cell-repellent material expressing repellency to cells as well as the bare surface of PDMS or glass. Because this method employed agarose as a cell repellent for cell patterning and no specified protein(s) for fabricating the cell culture surface for cell patterning, this would be a repellency-guided cell-patterning method.

Compared with micro-contact printing, which is one of the cell adhesive approaches, the advantage was thought to be basically compatible with the experimental conditions of conventional uniform culturing because of the repellency-based approach. On the other hand, in the case of micro-contact printing with specified protein(s), the concentration of protein solution as a printing ink, ink application, stamping force, and blocking method should be carefully considered.

Especially, in the case of neurons, axon guidance during a few days is important for the examination of axonal extension; ${ }^{9}$ therefore, the cast agarose stripes were for stripe assay with a sufficient maintenance time of at least 5 days. Furthermore, previously an agarose micro-structure was maintained for cell culturing in cell culture medium for 11 days. ${ }^{39}$

This method may be employed even by end-users without micro-fabrication facilities to make the PDMS molds, because the users can easily obtain the molds from micro-fabrication companies or through collaboration with engineering laboratories. Once the PDMS molds have been obtained, agarose casting requires users only to prepare an agarose solution and have a vacuum desiccator, both of which are commonly available in biological and medical laboratories. Furthermore, the users can repeatedly perform agarose casting with a single PDMS mold.

\section{Conclusion}

This study proposed a micro-molding method using viscous agarose gel solution. PDMS micro-channels were used as a mold and they were easily filled with agarose solution by the driving force of vacuum pressure accumulated in the PDMS forming the micro-channels without complex external pumping devices. As a result, a hydrogel patterning resolution of a few micrometers was achieved. Furthermore, this method was successfully applied to the micro-patterning of mammalian cells based on the repellency of the agarose pattern toward these cells. The authors believe that this method is user-friendly and useful not only for micro-molding but also for cellular patterning with the repellency of agarose without any special molecules for cell adhesion.

\section{Conflict of interest}

The authors have no conflict of interest directly relevant to the content of this article.

\section{Acknowledgements}

The study was partially supported by Bilateral Programs from the Japan Society for the Promotion of Science (JSPS) and JSPS KAKENHI Grant Number 25709081 (to Y. T.).

\section{References}

1 C. S. Chen, M. Mrksich, S. Huang, G. M. Whitesides and D. E. Ingber, Science, 1997, 276, 1425-1428.

2 S. N. Bhatia, U. J. Balis, M. L. Yarmush and M. Toner, FASEB J., 1999, 13, 1883-1900.

3 S. A. Ruiz and C. S. Chen, Stem Cells, 2008, 26, 2921-2927.

4 T. Takebe, K. Sekine, M. Enomura, H. Koike, M. Kimura, T. Ogaeri, R. Zhang, Y. Ueno, Y. Zheng, N. Koike, 
S. Aoyama, Y. Adachi and H. Taniguchi, Nature, 2013, 499, 481-484.

5 R. S. Kane, S. Takayama, E. Ostuni, D. E. Ingber and

G. M. Whitesides, Biomaterials, 1999, 20, 2363-2376.

6 N. Tanaka, H. Ota, K. Fukumori, J. Miyake, M. Yamato and T. Okano, Biomaterials, 2014, 35, 9802-9810.

7 D. Qin, Y. Xia and G. M. Whitesides, Nat. Protoc., 2010, 5, 491-502.

8 M. C. Berg, S. Y. Yang, P. T. Hammond and M. F. Rubner, Langmuir, 2004, 20, 1362-1368.

9 B. Knöll, C. Weinl, A. Nordheim and F. Bonhoeffer, Nat. Protoc., 2007, 2, 1216-1224.

10 S. Takayama, J. C. McDonald, E. Ostuni, M. N. Liang, P. J. Kenis, R. F. Ismagilov and G. M. Whitesides, Proc. Natl. Acad. Sci. U. S. A., 1999, 96, 5545-5548.

11 K. Y. Suh, J. Seong, A. Khademhosseini, P. E. Laibinis and R. Langer, Biomaterials, 2004, 25, 557-563.

12 K. Jang, K. Sato, K. Mawatari, T. Konno and K. Ishihara, Biomaterials, 2009, 30, 1413-1420.

13 K. Itoga, J. Kobayashi, M. Yamato, A. Kikuchi and T. Okano, Biomaterials, 2006, 27, 3005-3009.

14 T. Crouzier, H. Jang, J. Ahn, R. Stocker and K. Ribbeck, Biomacromolecules, 2013, 14, 3010-3016.

15 R. McBeath, D. M. Pirone, C. M. Nelson, K. Bhadriraju and C. S. Chen, Dev. Cell, 2004, 6, 483-495.

16 V. Vogel and M. Sheetz, Nat. Rev. Mol. Cell Biol., 2006, 7, 265275.

17 N. Idota, T. Tsukahara, K. Sato, T. Okano and T. Kitamori, Biomaterials, 2009, 30, 2095-2101.

18 E. A. Roth, T. Xu, M. Das, C. Gregory, J. J. Hickman and T. Boland, Biomaterials, 2004, 25, 3707-3715.

19 S. Iwanaga, Y. Akiyama, A. Kikuchi, M. Yamato, K. Sakai and T. Okano, Biomaterials, 2005, 26, 5395-5404.

20 H. Moriguchi, Y. Wakamoto, Y. Sugio, K. Takahashi, I. Inoue and K. Yasuda, Lab Chip, 2002, 2, 125-132.

21 A. Tourovskaia, T. Barber, B. T. Wickes, D. Hirdes, B. Grin, D. G. Castner, K. E. Healy and A. Folch, Langmuir, 2003, 19, 4754-4764.
22 C. H. Cho, J. Park, A. W. Tilles, F. Berthiaume, M. Toner and M. L. Yarmush, BioTechniques, 2010, 48, 47-52.

23 M. Mayer, J. Yang, I. Gitlin, D. H. Gracias and G. M. Whitesides, Proteomics, 2004, 4, 2366-2376.

24 M. M. Stevens, M. Mayer, D. G. Anderson, D. B. Weibel, G. M. Whitesides and R. Langer, Biomaterials, 2005, 26, 7636-7641.

25 J. R. Moffitt, J. B. Lee and P. Cluzel, Lab Chip, 2012, 12, 1487.

26 P. D. Benya and J. D. Shaffer, Cell, 1982, 30, 215-224.

27 V. Normand, D. L. Lootens, E. Amici, K. P. Plucknett and P. Aymard, Biomacromolecules, 2000, 1, 730-738.

28 Immediately Dangerous to Life or Health Concentrations (IDLH): Acrylamide-NIOSH Publications and Products, http://www.cdc.gov/niosh/idlh/79061.html, accessed May 2016.

29 K. Hashimoto, J. Sakamoto and H. Tanii, Arch. Toxicol., 1981, 47, 179-189.

30 C. M. Nelson, W. F. Liu and C. S. Chen, Methods Mol. Biol., 2007, 370, 1-10.

31 E. Y. Arashiro and N. R. Demarquette, Mater. Res., 1999, 2, 23-32.

32 A. F. Stalder, T. Melchior, M. Müller, D. Sage, T. Blu and M. Unser, Colloids Surf., A, 2010, 364, 72-81.

33 K. Tadakuma, N. Tanaka, Y. Haraguchi, M. Higashimori, M. Kaneko, T. Shimizu, M. Yamato and T. Okano, Biomaterials, 2013, 34, 9018-9025.

34 K. Shimba, K. Sakai, T. Isomura, K. Kotani and Y. Jimbo, Integr. Biol., 2015, 7, 64-72.

35 D. Juncker, H. Schmid, U. Drechsler, H. Wolf, M. Wolf, B. Michel, N. De Rooij and E. Delamarche, Anal. Chem., 2002, 74, 6139-6144.

36 K. Hosokawa, K. Sato, N. Ichikawa and M. Maeda, Lab Chip, 2004, 4, 181-185.

37 V. Ghetie and H. D. Schell, Experientia, 1971, 27, 1384-1385.

38 K. Hosokawa, M. Omata, K. Sato and M. Maeda, Lab Chip, 2006, 6, 236-241.

39 Y. Sugio, K. Komjima, H. Moriguchi, K. Takahashi and K. Yasuda, Sens. Actuators, B, 2004, 99, 156-162. 